\title{
Morphological and Molecular Characterization of Paragonimus skrjabini Complex from Yunnan, China: A Brief Report
}

\author{
Qiu-Hong Shu ${ }^{1} \cdot$ Shu-De $\mathrm{Li}^{2} \cdot$ Ming Tian ${ }^{2} \cdot$ Yong Meng ${ }^{1} \cdot$ Shu-Mei-Qi He ${ }^{2} \cdot$ Min Zhu $^{2} \cdot$ Miao-Miao Wang ${ }^{2}$. \\ Wen-Lin Wang ${ }^{2,3}$ (B)
}

Received: 13 May 2021 / Accepted: 4 August 2021 / Published online: 21 August 2021

(c) The Author(s) 2021

\begin{abstract}
Purpose To perform environmental sampling and molecular identification of Paragonimus in endemic regions, which may help in minimizing transmission among humans.

Methods Mountain crabs from the genus Potamiscus were collected and the encysted metacercariae were extracted and subjected to morphological identification, followed by animal inoculation in Sprague-Dawley (SD) rats. After 112 days of infection, animals were killed and adult worms were extracted from lungs and muscles. The morphology of adult worms was characterized by microscopy and molecular identification was done by polymerase chain reaction, followed by sequencing of coxl and ITS 2 genes. Phylogenetic analysis was done by maximum parsimony method.

Results A total of 447 crabs were captured from the streams of Tongchang Town, Jinping County, Yunnan Province, China. The infection rate was found to be $41 \%$ (186 out of 447 crabs). The metacercariae of Paragonimus skrjabini was identified by the characteristics round or spherical encysted form measuring 410 to $460 \times 400$ to $460 \mu \mathrm{m}$. After animal infection in SD rats, adults were presumptively confirmed to be P. skrjabini, which was also confirmed by gene amplification and sequence analysis of coxl and ITS2 regions. Paragonimus skrjabini clustered with previously reported $P$. skrjabini from Yunnan and Vietnam. The confidence values of their branches were $>95 \%$. Phylogenetic analysis of the ITS2 region revealed two distinct clusters with distinct geographical grouping. Phylogenetic analysis with the combined data sets reiterated the geographical grouping with $P$. skrjabini from Yunnan clustering with strains from Vietnam.
\end{abstract}

Conclusion Metacercariae of P. skrjabini was discovered in freshwater crabs in Yunnan province, China, and the strains were phylogenetically related to $P$. skrjabini from Vietnam.

Keywords P. skrjabini $\cdot$ Phylogenetic analysis $\cdot$ Paragonimiasis

Wen-Lin Wang

wenlinwang331@163.com

1 Department of Cardiology, The Second Affiliated Hospital of Kunming Medical University, No. 374, Dianmian Road, Kunming 651010, Yunnan, China

2 School of Basic Medical Sciences, Kunming Medical University, No. 1168, Chunrong West Road, Yuhua Street, Chenggong District, Kunming 65050, Yunnan, China

3 Department of Parasitology, Faculty of Basic Medicine, Kunming Medical University, No. 1168, Chunrong West Road, Yuhua Street, Chenggong District, Kunming 65050, Yunnan, China

\section{Introduction}

Paragonimiasis is an important food-borne zoonosis, especially in Asian countries. This parasitic infection manifests as acute or chronic lung infection caused by the trematodes of the genus Paragonimus. The genus Paragonimus consists of varied species such as Paragonimus skrjabini complex and the Paragonimus ohirai complex, overlapping the geographical ranges of the Paragonimus westermani complex [1]. The morphological features of large metacercariae found for the first time in Vietnam resembled that of Paragonimus skrjabini [2]. In Asia, P. skrjabini is an important pathogen, along with $P$. westermani, causing infections in humans throughout Asia. Among the different species complexes, $P$. skrjabini has been previously reported mainly in East Asia and China [3]. Initially thought to be a pathogen exclusively 
found in China, it has recently been reported from Japan, India, and Vietnam [4].

The life cycle of Paragonimus is relatively complex that requires a minimum of three hosts including a definitive host and two intermediate hosts [5]. The first and second intermediate hosts are frequently snails belonging to the families Assimineidae and Hydrobiidae and crabs belonging to the families Potamidae and Parathelphusidae [6]. Different species of Paragonimus have their own predilection for infecting specific genera of snails and crabs and hence the epidemiological prevalence of different species of Paragonimus is determined by the existence of suitable hosts. Some species of Paragonimus have been identified only from intermediate hosts in certain geographies that suggest infection and maintenance in non-human mammals [7, 8]. In Sichuan province, 18 species of freshwater crabs such as Stigmatomma. denticulatum, Sinopotamon. yaanense, Sinopotamon. davidi, and Haberma nanum were found to act as the second intermediate host [9].

The disease burden of paragonimiasis in China is the highest among all the countries due to dietary patterns. Nearly $80 \%$ of the Paragonimus species reported worldwide originated from China, which also has the highest disease burden in the world with $7.83 \%$ of prevalence of human paragonimiasis [10]. Also, China is considered as an ecological hotspot with high species diversity and endemicity of the snail and crab intermediate hosts of Paragonimus spp. Considering the vast landscape of China and the varied biotopes, the spread of specific species of Paragonimus is also presumed to be distinct in different provinces [10]. Yunnan is a province in southern part of China, which is more proximal to south-east Asian countries where P. heterotremus is the most prevalent causative organism of human paragonimiasis [6]. Although the incidence of paragonimiasis is high among the Chinese population, studies reporting the morphological and molecular characterization on P. skrjabini causing paragonimiasis are limited. In addition, the morphological characterization of Paragonimus species from non-mammalian hosts will help to understand the genetic variation and also to suggest suitable lifestyle modifications for populations at risk of paragonimiasis.

Here, we report the discovery of $P$. skrjabini metacercariae from mountain crabs from Yunnan Province, China. Adult worms were obtained by experimental infection of the metacercariae in experimental rats. The morphological and molecular relationships with other geographical populations in the P. skrjabini species complex are discussed further.

\section{Materials and Methods}

\section{Parasitological Methods}

The Paragonimus metacercariae were isolated from naturally infected mountain crabs with male crabs having hair on all eight legs and the presence of pointed umbilicus, belonging to the genus Potamiscus, the second intermediate hosts from Tongchang Town, Jinping County, Yunnan Province, China (Fig. 1). The sampling locations are mainly the moving streams of water without impeding vegetation. The morphological identification of the secondary hosts was done according to the classification method of "Chinese Medical Crustaceans" [11].
Fig. 1 Sampling distribution and previous reports of $P$. skrjabini

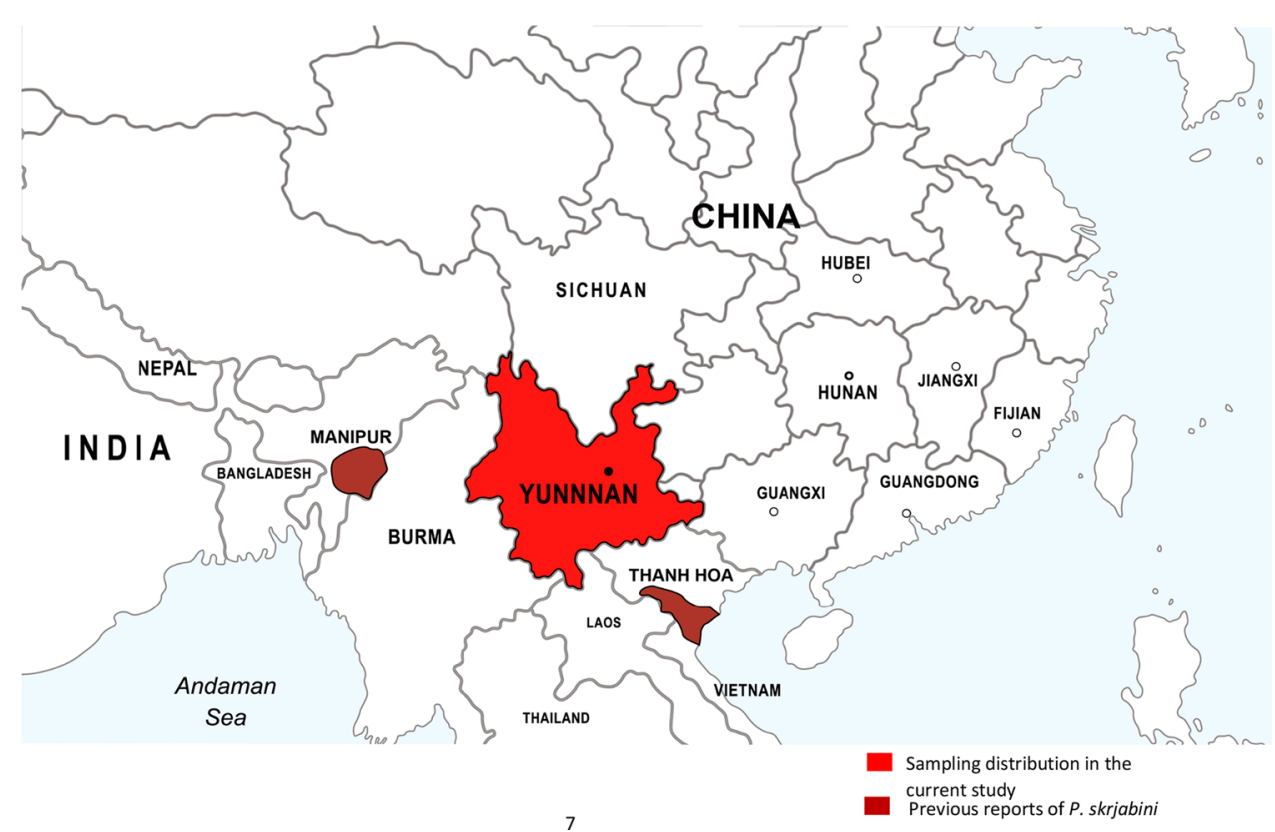


The crabs were crushed in a mortar, followed by sieving and washed with distilled water into a sedimentation cup with distilled water. The filtration was done with a filter of pore size $200 \mu \mathrm{m}$ and $1000 \mu \mathrm{m}$. The supernatant was discarded after $20 \mathrm{~min}$ and the same step was repeated four to five times. The sediment was then placed in a glass dish for microscopic biological observation. The metacercariae of Paragonimus were counted under the microscope and a part of the sediment with the metacercariae were fixed with absolute ethanol and stored in a refrigerator at $4{ }^{\circ} \mathrm{C}$ for molecular biological experiments.

\section{Experimental Infection}

Freshly isolated live metacercariae of Paragonimus (presumptively identified as $P$. skrjabini based on morphology) [12] were then injected intraperitoneally (15 metacercariae per rat) into paragonimiasis-negative SD rats (purchased from the Laboratory Animal Department of Kunming Medical University). All the animals were handled in accordance with the Guide for the Care and Use of Laboratory Animals published by the US National Institutes of Health (NIH Publication No. 8523, revised 1985). All experimental protocols were approved by the Animal Care and Use Committee of Kunming Medical University (reference: KMMU2015002). Experimental animal inoculation by subcutaneous injection was done, following which, five SD rats were killed on the $2 \mathrm{nd}$, 4 th, and 6 th week to confirm the infections of rats with adult Paragonimus spp. After 114 days of injection, the SD rats were dissected to isolate the cysts, eggs, and adult worms from the muscles, abdomen, liver, thoracic cavity, and lungs of SD rats. The isolated adult worms were used for genomic DNA extraction and for preparing permanent slides for microscopic confirmation by fixing them on to glass slides with alcohol, formalin, and acetic acid.

\section{Morphologic Identification}

The morphologic features of the cysts, metacercariae, and adults were used for the presumptive identification of $P$. skrjabini. The microscopic and morphological characterization was based on the arrangement of spines on the epidermis of the adult worms, the degree of branching of the ovaries and testes, the aspect ratio and relative size of the suction discs, the characteristics of the cyst walls (presence or absence, number and thickness), the relative diameter of the suction discs, the anterior extent of the excreted bladder, the length of the probe on the mouth suction disc, the presence of pigmented granules in the body, the number of flame cells, body spine and the alignment of the nipples around the discs [13].

\section{Molecular Analysis}

Genomic DNA was extracted from both the adult worms from SD rats and also from the metacercariae from crabs using QIAamp DNA Mini kit (QIAGEN, Hilden, Germany). The whole process was carried out in strict accordance with the instructions of the manufacturer. The final elution of DNA was done with $100 \mu \mathrm{L}$ of distilled water. The extracted total genomic DNA was quantified and stored in the refrigerator at $-20{ }^{\circ} \mathrm{C}$ until further use.

\section{Polymerase Chain Reaction Amplification of cox1 and ITS2}

Polymerase chain reaction (PCR) was performed with primers targeting a fragment of the partial mitochondrial cytochrome oxidase subunit 1 ( cox 1$)$ gene and the nuclear ribosomal second internal transcribed spacer (ITS2) region synthesized by Shanghai Bioengineering Co., Ltd. The primers used for amplifying coxl gene fragments were COIF-5'-GAGGTGTATGTCCTGATTTTGCC-3' and COIR - 5'-GACCTCACCCAATGACCCTGCAACA-3' and the primers for amplifying ITS2 gene fragments were ITS2F-5'-GGGTACCGGATCACTCGCTCGGTG-3' and ITS2R-5'-GGGGATCCTGGTTGCCTTAGTCTC CGC-3' [14]. PCR was performed in $25 \mu \mathrm{L}$ volume with 2 $\mu \mathrm{L}$ of template DNA corresponding to $0.1 \mathrm{ng}$ and $1 \mu \mathrm{L}$ of primers $(10 \mu \mathrm{mol} / \mu \mathrm{L}), 2.5 \mu \mathrm{L}$ of $10 \times$ PCR buffer, $1 \mu \mathrm{L}$ of $10 \mathrm{mM}$ deoxynucleotide triphosphates (dNTP), $0.1 \mu \mathrm{L}(0.5$ units) of Taq enzyme $(5 \mathrm{U} / \mu \mathrm{L})$ and $17.4 \mu \mathrm{L}$ of PCR grade water. The PCR amplification was set up in an ice bath and nducted in the TaKaRa PCR instrument (Bao Biology Co., Ltd.) and the amplification conditions were as follows: initial denaturation of $95^{\circ} \mathrm{C}$ for $3 \mathrm{~min}$, followed by 35 cycles of denaturation at $93{ }^{\circ} \mathrm{C}$ for $1 \mathrm{~min}$, annealing at $48{ }^{\circ} \mathrm{C}$ (for $\operatorname{cox} 1$ ) $/ 60{ }^{\circ} \mathrm{C}$ (for ITS2) for $1 \mathrm{~min}$ and extension at $72{ }^{\circ} \mathrm{C}$ for $1 \mathrm{~min}$, followed by final extension at $72{ }^{\circ} \mathrm{C}$ for $5 \mathrm{~min}$. The expected length of the PCR fragments was 500-750 base pairs. The detection of PCR-amplified products was done by agarose gel electrophor-esis with $1.5 \%$ agarose gel immersed in $1.0 \%$ Tris-ACETATEEDTA buffer stained with ethidium bromide. The purity and quantity were estimated using the gel documentation system imaging technique (BioRad Laboratories).

The PCR products were then subjected to bidirectional sequencing using the same PCR primers by Shanghai Biotechnology Co., Ltd (Hitachi fluorescent DNA sequencer SQ-3000). The forward and reverse sequences were then manually curated and aligned with the DNASTAR v7.1 software and the consensus sequence were used for bioinformatic analysis.

The initial quality check of the sequences was done by checking the coverage and alignment with previously 
submitted sequences in National Center for Biotechnology Information (NCBI) using the BLAST tool. Previously submitted sequences of cox 1 and ITS2 were retrieved from $\mathrm{NCBI}$ and compared with the sequences obtained in this study with ClustalX software with default parameters. A phylogenetic analysis was done by the maximum parsimony (MP) method as per the Kimura 2-parameter model. Neighbor-joining method (NJ) was used for the ML heuristic method with bootstrap replicates of 1000 . The analysis was performed with MEGA5.0 software [15]. The cutoff value for consensus tree was set to $75 \%$.

\section{Results}

\section{Morphological Identification}

\section{Metacercariae and Adult Worms}

A total of 447 crabs were captured from the streams of Tongchang Town, Jinping County, Yunnan Province, China, and were included for this study. The infection rate was found to be $41 \%$ ( 186 out of 447 crabs). Out of the 186 crabs, a total of 551 metacercariae were isolated and identified (range: 1 to 100, mean: 2.96 per crab).

The metacercariae of $P$. skrjabini were identified by the characteristic round or spherical encysted form measuring 410 to $460 \times 400$ to $460 \mu \mathrm{m}$. The encysted form had three layers (cyst walls) with an outermost thin layer, which could be easily ruptured, a thicker middle layer and a thinner inner layer. The average thickness of the outer and inner wall was found to be 4 and $12 \mu \mathrm{m}$, respectively. The encysted metacercariae were excysted by mechanical shearing and the excysted metacercariae measured 650 to $670 \times 370$ to $420 \mu \mathrm{m}$. The oral and ventral suckers were $80-120 \mu \mathrm{m}$ and $120-60 \mu \mathrm{m}$, in diameter, respectively. These features roughly correlated with $P$. skrjabini and the metacercariae were subjected to animal inoculation. After experimental infections, two to four adult worms were retrieved from each animal from the lungs and muscles. The dimensions of the adult worms ranged from 950 to $1250 \mu \mathrm{m} \times 400$ to $500 \mu \mathrm{m}$ with an oral sucker measuring 460 to $530 \mu \mathrm{m} \times 625$ to $710 \mu \mathrm{m}$. The diameter of the ventral sucker ranged from 700 to $800 \mu \mathrm{m}$. The other characteristic features observed were in the left ( 660 to $2000 \times 320$ to 1120$)$ and right testis (1600 to $1650 \mu \mathrm{m} \times 885$ to $1175 \mu \mathrm{m})$ and an ovary (1575 to $1800 \mu \mathrm{m} \times 1325$ to $1500 \mu \mathrm{m})$. Based on these morphologic features, worms that were presumptively identified as $P$. skrjabini were subjected to molecular identification.

\section{Molecular Identification}

PCR amplification of the coxl and ITS2 regions, followed by agarose gel electrophoresis, revealed amplicons of about 500 and 750 bp in length, respectively. BLAST searches using our sequences as queries found matches with $100 \%$ coverage in GenBank.

\section{Phylogenetic Analysis}

Based on sequence similarity, a total of 8 P. skrjabini and the related $P$. miyazakii sequences were downloaded from NCBI and used for phylogenetic analysis (Table 1).

In the phylogenetic tree of COI gene (Fig. 2), P. skrjabini clustered with previously reported $P$. skrjabini from Yunnan and Vietnam. The confidence values of their branches were $>95 \%$ (Fig. 2). Phylogenetic analysis of the ITS2 region (Fig. 3) revealed two distinct clusters with distinct geographical grouping. Phylogenetic analysis with the combined datasets (Fig. 4) reiterated the geographical grouping with $P$. skrjabini from Yunnan clustering with strains from Vietnam.

Table 1 DNA sequences of Paragonimus spp. used for the phylogenetic analysis

\begin{tabular}{|c|c|c|c|c|c|c|}
\hline \multirow{2}{*}{$\begin{array}{l}\text { Serial } \\
\text { number } \\
\text { (No.) }\end{array}$} & \multirow[t]{2}{*}{$\begin{array}{l}\text { Generic name } \\
\text { (generic name) }\end{array}$} & \multirow[t]{2}{*}{$\begin{array}{l}\text { Species name } \\
\text { (specific name) }\end{array}$} & \multicolumn{2}{|c|}{$\begin{array}{l}\text { GenBank serial number } \\
\text { (accession number) }\end{array}$} & \multirow[t]{2}{*}{$\begin{array}{l}\text { Sampling point } \\
\text { (location of sample) }\end{array}$} & \multirow[t]{2}{*}{$\begin{array}{l}\text { Specimen number } \\
\text { (sample code)* }\end{array}$} \\
\hline & & & $\operatorname{cox} 1$ & ITS2 & & \\
\hline 1 & Paragonimus & miyazakii & AY618807 & AY618757 & Japan (Japan) & \\
\hline 2 & Paragonimus & miyazakii & AY618834 & AY618742 & Fujian, China (Fujian Province, China) & \\
\hline 3 & Paragonimus & skrjabini & AB703456 & AB703448 & Vietnam & \\
\hline 4 & Paragonimus & skrjabini & AY618759 & AY618729 & Guangxi, China (Guangxi Province, China) & \\
\hline 5 & Paragonimus & skrjabini & AY618760 & AY618743 & Sichuan, China (Sichuan Province, China) & \\
\hline 6 & Paragonimus & skrjabini & AY618763 & AY618748 & Hubei, China (Hubei Province, China) & \\
\hline 7 & Paragonimus & skrjabini & AY618801 & AY618730 & Guangdong, China (Guangdong Province, China) & \\
\hline 8 & Paragonimus & skrjabini & AY618805 & AY618734 & Yunnan-China (Yunnan Province, China) & \\
\hline 9 & Paragonimus & skrjabini & MN650827.1 & - & Yunnan, China (Yunnan Province, China) & Sample28 \\
\hline
\end{tabular}

*Sequences obtained inthe study 
Fig. 2 Phylogenetic tree by maximum parsimony method for COI gene

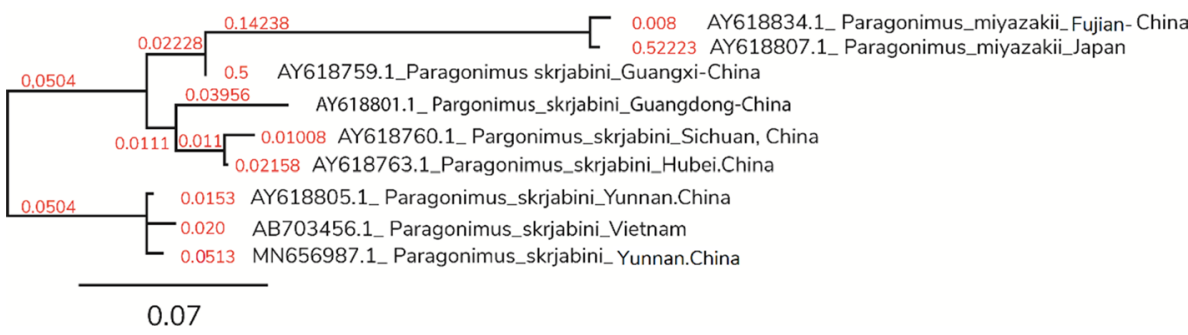

Fig. 3 Phylogenetic tree by maximum parsimony method for ITS2 gene

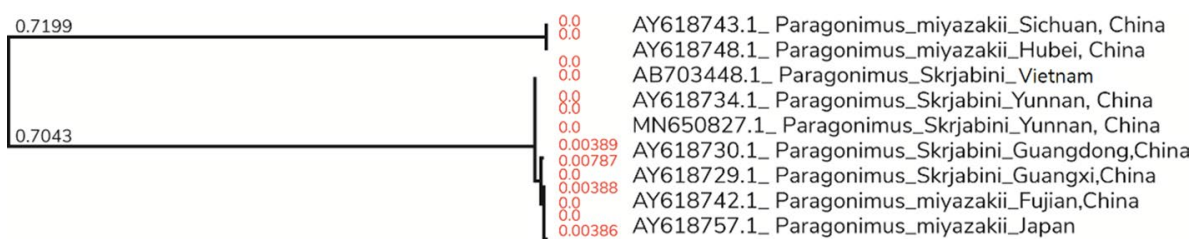

0.4

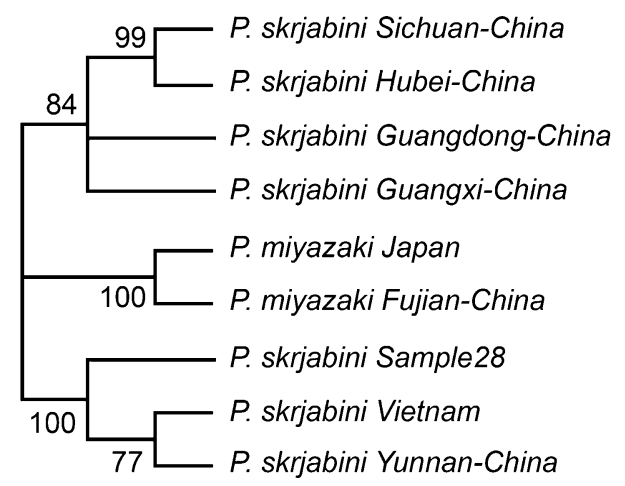

Fig. 4 Phylogenetic tree by maximum parsimony method with both COI and ITS2 regions

\section{Discussion}

China is endemically diverse with respect to Paragonimus species, wherein 38 species of Paragonimus have been reported so far $[10,16]$. Considering the diverse landscape and availability of suitable hosts, a stringent geographic predilection for occurrence is presumed in China [8]. In this study, we assessed the occurrence of a medically important species of the genus Paragonimus, P. skrjabini, and evaluated the phylogenetic relationship to substantiate the geographical predilection for occurrence.

Often, the differential morphological features of metacercariae are not well marked; hence, metacercariae stage from the secondary intermediate hosts [6] and adults from the mammalian hosts are used for morphological identification. In this study, morphological identification made at the metacercariae and adult stages were concordant with each other, further substantiating the role of morphological identification. The morphological features of the metacercariae and adult worms in the present study resembled that of $P$. skrjabini, where the adult worm was initially described from the palm civet Paguma larvata in Guangzhou City, Guangdong Province, China, and subsequently infections in humans were also reported from the same province $[17,18]$. Previously, morphological identification-based reporting was published from Manipur, India, by Singh et al., but molecular identification was not performed [4].

The infection rate by $P$. skrjabini in our study was attributed to $41 \%$ (186 out of 447 crabs). Similarly, in a study conducted in Sichuan province, the average rates of freshwater crab infection by metacercariae of $P$. skrjabini were 81\% (S. denticulatum, Nantong), 62.5\% (S. davidi, Shizhu), 63.2\% (S. nanum, Fengjie), and 88.5\% (S. davidi, Xingwen), respectively [9]. A study of $P$. skrjabini in Three Gorges Reservoir Region showed that the rate of freshwater crab infections with P. skrjabini metacercariae was $39.65 \%$ (1053/2656).

ITS2 and cox 1 sequences have been widely used in intraspecific variation study in Paragonimus species [19-21]. The phylogenetic analysis utilizing the sequence variation in the ITS2 and coxl genes revealed interesting phylogenetic features. While coxl phylogenetic analysis revealed distinct clustering of the related subspecies, $P$. miyazakii. In the ITS phylogeny, $P$. miyazakii clustered with $P$. skrjabini isolated from Sichuan, Fujian, Hubei, and Guangxi. P. miyazakii is predominantly reported from Japan, whereas $P$. skrjabini is predominantly reported from southeast Asia including Vietnam. Fujian, Hubei, and Sichuan provinces are geographically inclined towards east Asia. While Yunnan is proximal to the other South-east Asian countries. Further, in a previous study on P. skrjabini from Vietnam, the majority of the strains clustered with those found in Yunnan province, while P. miyazakii clustered with 
P. skrjabini from Fujian [22]. The phylogenetic similarities of $P$. skrjabini from Yunnan and Vietnam were also substantiated by the phylogenies derived from the combined dataset wherein strains from Yunnan and Vietnam clustered together. Notably, P. skrjabini species from Vietnamese and India population, together with the Chinese Yunnan population, form a distinct clade within the P. skrjabini complex. Further, a study by Blair et al. reported that coxl gene sequences from P. skrjabini and P. miyazakii revealed a number of differences, whereas ITS2 gene sequences were identical between P. skrjabini and P. miyazakii. We speculate that ITS2 gene is useful for the identification of Paragonimus strains, while coxl gene is more suitable for Paragonimus strains typing [23].

In conclusion, we discovered metacercariae of $P . s k r$ jabini in freshwater crabs in Yunnan province, China, and the strains were phylogenetically related to $P$. skrjabini from Vietnam, revealing a possible route of spread.

Funding The work was funded by Yunnan Provincial Department of Science and Technology Kunming Medical University grant for applied basic research (Project No.: 2019FE001 (-161). Proteomics study of paragonimiasis induced lung injury in rats based on iTRAQ Technology. General project of Yunnan Provincial Science and Technology Department Kunming Medical University appliedbasic research (Project No.: 202001AY070001-058).

Open Access This article is licensed under a Creative Commons Attribution 4.0 International License, which permits use, sharing, adaptation, distribution and reproduction in any medium or format, as long as you give appropriate credit to the original author(s) and the source, provide a link to the Creative Commons licence, and indicate if changes were made. The images or other third party material in this article are included in the article's Creative Commons licence, unless indicated otherwise in a credit line to the material. If material is not included in the article's Creative Commons licence and your intended use is not permitted by statutory regulation or exceeds the permitted use, you will need to obtain permission directly from the copyright holder. To view a copy of this licence, visit http://creativecommons.org/licenses/by/4.0/.

\section{References}

1. Blair D, Nawa Y, Mitreva M, Doanh PN (2016) Gene diversity and genetic variation in lung flukes (genus Paragonimus). Trans R Soc Trop Med Hyg 110:6-12

2. Doanh PN, Shinohara A, Horii Y, Habe S, Nawa Y, The DT, Le NT (2007) Morphological and molecular identification of two Paragonimus spp., of which metacercariae concurrently found in a land crab, Potamiscus tannanti, collected in Yenbai Province. Vietnam Parasitol Res 100:1075-1082

3. Sanpool O, Intapan PM, Thanchomnang T, Janwan P, Nawa Y, Blair D, Maleewong W (2013) Molecular variation in the Paragonimus heterotremus complex in Thailand and Myanmar. Korean J Parasitol 51:677-681

4. Singh ST, Singh DL, Sugiyama H (2006) Possible discovery of Chinese lung fluke, Paragonimus skrjabini in Manipur, India. Southeast Asian J Trop Med Public Health 37(Suppl 3):53-56

5. Yoshida A, Doanh PN, Maruyama H (2019) Paragonimus and Paragonimiasis in Asia: an update. Acta Trop 199:105074
6. Blair D, Xu ZB, Agatsuma T (1999) Paragonimiasis and the genus Paragonimus. Adv Parasitol 42:113-222

7. Doanh NP, Tu AL, Bui TD, Loan TH, Nonaka N, Horii Y, Blair D, Nawa Y (2016) Molecular and morphological variation of Paragonimus westermani in Vietnam with records of new second intermediate crab hosts and a new locality in a northern province. Parasitology 143:1639-1646

8. Song J-H, Dai F, Bai X, Kim T-I, Yang H-J, Kim T-S, Cho S-H, Hong S-J (2017) Recent incidence of Paragonimus westermani Metacercariae in freshwater Crayfish, Cambaroides similis, from two Enzootic Sites in Jeollanam-do, Korea. Korean J Parasitol 55:347-350

9. Liu J, Liu Z, Luo X, Yi D, Gu G, Liu C (1992) Study on distribution and natural infection of crabs containing Paragonimus metacercariae in Sichuan. Sichuan J Parasitic Dis 20:25-31

10. Liu Q, Wei F, Liu W, Yang S, Zhang X (2008) Paragonimiasis: an important food-borne zoonosis in China. Trends Parasitol 24:318-323

11. Aiyun D (1984) Chinese medical Crustaceans. Science Press

12. Blair D (2019) Paragonimiasis. Adv Exp Med Biol 1154:105-138

13. Blair D, Chang Z, Chen M, Cui A, Wu B, Agatsuma T, Iwagami M, Corlis D, Fu C, Zhan X (2005) Paragonimus skrjabini Chen, 1959 (Digenea: Paragonimidae) and related species in eastern Asia: a combined molecular and morphological approach to identification and taxonomy. Syst Parasitol 60:1-21

14. Bowles J, Blair D, McManus DP (1995) A molecular phylogeny of the human schistosomes. Mol Phylogenet Evol 4:103-109

15. Lou HQ, Hu Y, Zhang L, Zhang YJ, Tu PG, Yu XT, Jin YJ, Wang L (2011) Epidemiological survey and phylogenetic analysis of Paragonimus westermani isolates in Jinhua. China Afr J Biotechnol 10:14286-14292-14292

16. Zhang X, Wang Y, Wang G, Chen W, He X, Niu H, Li Z, Chen L, Wang L (2012) Distribution and clinical features of Paragonimiasis skrjabini in Three Gorges Reservoir Region. Parasitol Int 61:645-649

17. Chen H-T (1958) The occurrence of a new type of Paragoni$m u s$ and some clinical problems related to lung flukes in China. Guangzhou 1959:192-193

18. Chung H-L, Tsao W-C (1962) Paragonimus westermani (Szechuan variety) and a new species of lung fluke: Paragonimus szechuanensis. Part II. Studies on the clinical aspects of Paragonimus szechuanensis: a new clinical entity. Chin Med J 81:419-434

19. Lee S-U, Huh S (2004) Variation of nuclear and mitochondrial DNAs in Korean and Chinese isolates of Clonorchis sinensis. Korean J Parasitol 42:145-148

20. Nagaraja null, Nagaraju J, Ranganath HA, (2004) Molecular phylogeny of the nasuta subgroup of Drosophila based on 12S rRNA, $16 \mathrm{~S}$ rRNA and CoI mitochondrial genes, RAPD and ISSR polymorphisms. Genes Genet Syst 79:293-299

21. Park G-M, Im K-I, Yong T-S (2003) Phylogenetic relationship of ribosomal ITS 2 and mitochondrial COI among diploid and triploid Paragonimus westermani isolates. Korean J Parasitol 41:47-55

22. Doanh PN, Hien HV, Nonaka N, Horii Y, Nawa Y (2013) Discovery of Paragonimus skrjabini in Vietnam and its phylogenetic status in the Paragonimus skrjabini complex. J Helminthol $87: 450-456$

23. Blair D, Wu B, Chang ZS et al (1999) A molecular perspective on the genera Paragonimus Braun, Euparagonimus Chen and Pagumogonimus Chen. J Helminthol 73:295-299

Publisher's Note Springer Nature remains neutral with regard to jurisdictional claims in published maps and institutional affiliations. 